\title{
Nurses' pain management competency and associated factors among nurses working in public hospitals, jimma zone, oromia regional state, southwest Ethiopia
}

\begin{abstract}
Background: Pain management for hospitalized patient requires nurses' pain management competency. However; there is paucity of information on nurse's pain management competency in Jimma Zone public hospitals.

Objective: To assess nurses' pain management competency and associated factors among nurses working in public Hospitals, Jimma zone, Oromia Regional State, South west Ethiopia.

Methods: Institutional based cross- sectional study design was conducted on 310 nurses working in public hospitals, Jimma zone, from March 1-28, 2017. The study subjects were selected by simple random sampling technique using lottery method. Data was collected using standardized self-administered questionnaire. SPSS 20.0 version was used for data entry and analysis. Variables with $\mathrm{p}<0.25$ in the bivariate analyses were entered into a multivariable regression analysis to identify the independent factors associated with nurses' competency. Significant factors reported at $\mathrm{P}<0.05$.

Results: Respondents who were working at medical with $[A O R=2.05(1,02,4.12)]$, Nurses' who were working at surgical ward with $[A O R=0.19(0.05,0.64)]$ and Nurse-Physician work relationship with $[\mathrm{AOR}=2.36(95 \% \mathrm{Cl}: 1.36,4.08)]$ significantly associated with nurses' pain management competency.

Conclusion and Recommendation: The overall nurses' pain management competency level in Jimma zone, Public hospital is very low. Among many factors contributed to the nurses' patient pain management competency were nursephysician work relationship, presence of protocol and working unit. Those show that Jimma public hospitals should design strategy to improve pain management competency. Nurses' pain management competency enhances utilization of protocols, that specifies pain management by unit and promote nurse-physician relation.
\end{abstract}

Key words: Competency, pain management, nurses', jimma zone, public hospitals

\section{Introduction}

Even though pain is defined in many ways the most popular one is IASP definition which is an unpleasant sensory and emotional experience associated with actual or potential tissue damage or described in terms of such damage $[1,2]$. Based on duration pain classified as acute or chronic or breakthrough (pain in controlled pain). Acute pain is rapid but diminishes under treatment. Chronic pain becomes progressively worst and reoccurs intermittently. Clinical manifestation of pain is a highly subjective and deeply personal experience. Patient may have as difficulty of concentrating, lack of energy, lost productivity, decreased quality of life and inability to complete daily tasks [3].
Pain management is the alleviation of pain or a reduction in pain to a level that is acceptable to the client by pharmacologic and non-pharmacologic. Pain management is an important aspect of nursing care to promote healing, prevent complications and reduce suffering [4]. The level of pain relief depends on health care provider's competency to manage pain. Pain management competency refers to the understanding and practices of pain management domains:-multidimensional nature of pain, pain assessment and measurement, pain management and clinical condition of pain [5]. These domains of pain management competencies address the fundamental concepts and complexity of pain management competency and aligned

\section{Abiru Neme*, Gugsa Nemera and Gadisa Bekele}

Department of Nursing, Jimma University, Ethiopia

*Author for correspondence:

abiruneme@gmail.com 
with the outline categories of the International Association for the Study of Pain management $[6,7]$.

Unrelieved or undertreated pain can negatively affect an array of person's quality of life, including increasing functional impairment and disability, psychological distress (anxiety, depression) and sleep deprivation [8,9]. Approximately 116 million Americans suffer from chronic pain alone [10]. Likewise more than 25 million people experience acute pain as a result of injury or surgery.

In Australia, it is estimated that one in five people (about 3.2 Million Australians) including children and adolescents, was suffer chronic pain in their lifetime [11]. In Ethiopia, a study conducted by the Ethiopian Public Health Association in 2013 showed that health care providers believe that pain was unmanaged due to lack of training and education, adequate absence of medications, poor knowledge and attitude among professionals lead to unreasonable fear of side-effects or addiction [12].

Factors affecting management of pain competency include: lack of nursephysician collaboration, nurse related factors, organizational factors and nurse pain management domain. Nurses' pain management competency concerns how nurses manage pain. There was increased focus on pain management programs and the development of new standards for pain assessment and management. Ward nurses have a central role in pain management competency. It is ward nurses who spend more time with patients and have the responsibility for assessing patient's pain intensity, administering prescribed analgesic treatments and monitoring side effect of drugs [13].

Pain management competency is the most fundamental part of the nurses' responsibility [14]. To indicate gap, there is no such study done on pain management competency in Ethiopia.

According to the American Nurses Association (ANA), the nurses' role in pain management includes the entire nursing process, assessment of pain, plan of pharmacologic and non-pharmacologic pain management strategies, implementation and evaluation of the response of the patient to the interventions [15]. The competencies address the fundamental concepts and complexity of pain; how pain is observed; collaborative approaches to treatment options and application of competencies in the context of various settings, populations and care teams [16-20].

Staff work relationship among NursePhysician is the important part of pain management competency. Nurse-Physician pain management competency described as "actions related to sharing information about pain management, participating in decision-making concerning pain management and providing comprehensive care to patients from a patientcentered pain [21]. Lack of collaboration on pain management was the most that affect pain management competency of nurses'. From the study conducted in Japan indicated that, three factors on pain management between nursephysician: sharing of patient information on pain management, joint participation on pain management and cooperativeness on pain management [22].

Nurses' had the opportunity to discuss matters with a physician as they arose, arrangements for formal, regular clinical supervision sessions were not always in focused on pain management competency[23].

Some personal factors of nurses often contribute to nurse pain management competency. A nurses' past personal experience of pain and medication use has been found to be an importantfactorinchangingtheirattitudetowards pain management. This helps nurse to achieve optimal pain management outcome in their pain management competency. For example, study conducted in North-eastern United States indicated that $64.5 \%$ who answered yes to having had past personal pain experience that required medication or treatment [24].

Age, Sex, working unit, graduated institution may affect nurses' pain management competency. Nurses' who obtained their education from government have high competency on pain management than private institution.

Nurse related factor for pain management competency include. Study conducted in Bangladesh indicated $63.4 \%$ of the nurses never read nursing journals, $26.8 \%$ did yearly, $5.4 \%$ did quarterly and 3.2\%did it monthly. The nurses' pre-service training on pain management has led to improve pain relief for patients [25]. Another study conducted in Bangladesh 
indicates that majority of the nurses had never had training on; pain assessment methods and tools $(72.9 \%)$ [26].

Organizational factors like presence of Protocol and guidelines, medication availability, support and feedback on pain management can affect pain management. The study conducted in Ireland, indicated more than half $(57.4 \%)$ of the nurse always used a pain assessment tool for pain management for their pain management competency [27]. Another study also done in Ireland indicated that $38.3 \%$ respondent reported that they used a pain assessment tool frequently, with the remaining $4.3 \%$ of respondents rarely used pain assessment tool for their pain management competency [28].

For pain management, having pain protocol is most important. Study conducted in Bangladesh revealed that majority of the nurses $(59.1 \%)$ stated that there was no pain management standard or protocol used as a basis in the hospital [29]. Pain management guidelines are essential regardless of the availability of well-defined guidelines to help nurses understand management of pain [30] Medication availability is the most important for nurses' pain management competency. World Health Organization (WHO) uses morphine consumption statistics as a broad indicator of progress to improve pain relief. In 2003 Ethiopia had one of the lowest morphine /capita consumption $0.0005 \mathrm{mg}$ compared with the global mean of $5.85 \mathrm{mg}$. Again in 2006, the consumption was reported as $0.0002 \mathrm{mg} /$ capita; Uganda $0.3136 \mathrm{mg} / \mathrm{capita}$; Mali $0.0181 \mathrm{mg} / \mathrm{capita}$; Sudan 0.0230 Kenya; $0.1292 \mathrm{mg} /$ capita [31].

According to study conducted in Bangladesh most of the participants, $91.1 \%$ had not read any guidelines for pain management competency [19]. The study conducted on perceived clinical competency among nursing in Gondar referral hospital indicated that $48.7 \%$ of the respondents clinically competent on management of patient [32].

So, the reason for this study in generally, may lie in the scant interest and attention of nurses regarding pain management competency and factors affecting pain management competency of nurses'.

\section{Methods and Materials}

The study was conducted from March 1-March 28, 2017. Institutional based cross sectional study was conducted among nurses working in public hospitals, Jimma zone,
(JUMC, Agaro, Seka Chekorsa, Limu Genet and Shenen gibe). Wards were selected in cooperation with nursing head of each hospital. The following hospital departments were included: Medical unit, surgical unit, ICU unit, Emergency, Gynecology and obstetrics ward, pediatric ward, Psychiatric ward and other wards (neonatology, NICU, Stroke, OR).

\section{- Study design}

Institutional based cross sectional study was conducted

\section{- Populations}

\section{- Source population}

The source populations were all nurses in public hospitals, Jimma zone

\section{- Study population}

The study population was nurses who were working in public hospitals, Jimma zone

\section{- Inclusion and exclusion criteria}

\section{- Inclusion criteria}

Nurses' who were working in wards and available during data collection time

\section{- Exclusion criteria}

Nurses who were on annual leave

Sick leave during data collection period

\section{- Sample size and sampling technique}

Sample size was determined using single population proportion formula by considering, 95\% confidence level and 0.05 margin of error. $\mathrm{p}=0.5$ (in the absence of a similar previous study and to achieve the maximum possible sample size.

$$
n=\left(\frac{z \alpha}{2}\right)^{2} 0.5(1-0.5) /(0.05)^{2}=384
$$

Since the total population was less than 10000, Correction formula was used and minimum sample size was obtained.

$\mathrm{Nf}=\mathrm{N} \times \mathrm{n} / \mathrm{n}+\mathrm{N}=703 \times 384 / 384+703=248$

Considering $25 \%$ non-response rate $=248 \times$ $25 \%=62$

$\mathrm{nf}=248+62=310$

Finally $=310$ nurses' were included in the study

The total numbers of nurses (703) in the hospitals were considered and taken. First, total sample size (310) were estimated based on the 
total number of nurses' who were working in Jimma public hospitals. Next the determined sample was proportionally allocated to each hospital. The proportional allocation for each hospital are as follow: JUMC (558), Agaro [32], Seka chekorsa [32],Limmu Genet and Shenen Gibe. Then the sample proportionally allocated to each hospitals. Finally nurses' who were working during study period and fulfill the inclusion criteria were selected by simple sampling technique, lottery method was used to select.

Pain management Competency: Nursing competency score of pain management assessed using 39 questions developed by Margo McCaughey [33]. Each correct answer recorded as 1 and the incorrect answer recorded as 0 and entered into computer. Total score computed and converted to $100 \%$. Those study subjects who scored $50 \%$ and above are regarded as competent, whereas those who scored below $50 \%$ are regarded as in competent.

The data was collected by self-administered structured questionnaire which have five parts.

Part I: Socio demographic variables, Part two: Nurse Related Factor's questions, Part three: Staff Work Relationship (NursePhysician) questions, Part four: questions related to Organizational Factor, Part Five: Nurse Pain Management Competency Associated Factors

The tools were developed and taken after intensive review of literature and American Pain Society, the World Health Organization and the National Comprehensive Cancer Network Pain Guidelines [22,23,33]. The tool was developed in 1987 and has been used extensively from 1987- present [33]. The tool has been revised over the years to reflect changes in pain management practice. Content and construct validity have been established. The content of the KASRP was derived from pain management guidelines and standards including those of American Pain Society, The World Health Organization (WHO) and the U.S. Agency for Health Care Policy and Research. The KASRP has well established psychometric properties. It has been recommended to avoid distinguish items a measuring either knowledge or attitudes due to the overlap in some item and to report the percentage of correct responses. This tool also used by different country and Ethiopia on pain management [34].

Training was given for data facilitator by the principal investigator to make them familiar with the data collection tool. After that, based on their willingness to participate, questionnaire was distributed among nurses and clarification for any difficulty was the duty of the data facilitator and principal investigator. Principal investigator was assisted and the data facilitator as well as the participant nurses during data collection. Data was collected from nurses who were working in Jimma public hospitals. Finally completed questionnaire was returned to the supervisor.

Data cleaned and checked for its completeness. SPSS 20.0 version for windows was used for data entry and analysis. Descriptive analyses were performed on all study variables. Binary logistic regression analyses used to see the strength of association between independent variables and dependent variable. Variables with $p \leq 0.25$ in the bivariate analyses were entered into a multivariable logistic regression analysis to identify the independent factors associated with nurses' competency. Significant factors reported at $\mathrm{P}<0.05$. Results were presented in frequency, tables and charts.

The questionnaires were pre tested and data facilitators were trained for two day on the objectives of the study, sampling procedure and checking the completeness of questionnaires. The data were facilitated by 8 B.Sc nurses having previous experience in data supervision. Continuous follow-up and supervision was performed by principal investigator throughout the data collection period. The collected data was checked for completeness before data entry.

\section{Ethical Consideration}

Before data collection, ethical clearance was obtained from IRB committee, Jimma University. Formal letter of cooperation was written to respective administrators. Confidentiality maintained at all levels of the study by not writing the respondents name. Respondent's involvement in the study was on a voluntary basis; respondents who are unwilling to participate in the study and those who wish to quit their participation at any stage informed to do so without any restriction.

\section{Dissemination of the Study Result}

The study result copies was given to Jimma university, School of Post graduate, School of nursing and Midwifery. In addition, dissemination could facilitated through public outreach like presentation in association, 
different conference and to those who are in need of these results and accordingly will advocate for those who can implement it, example to the Ministry of Health, Hospitals and health centre administration for the arrangement of some training for their particular staffs. Finally the manuscript will be submitted to scientific journals for possible publication.

\section{Results}

\section{- Socio demographic}

Two hundred and ninety eighty respondents completely responded, producing a response rate of $(96 \%)$. Of total respondents 177 (59.2\%) were male, $205(68.8 \%)$ were in age range of 36-55 years, 128 (42.8\%) orthodox by religion, $181(61.5 \%)$ were bachelor degree holders, 146 (48.8\%) of them were single, 146 (48.8\%) married regarding their marital status, 236 (79.2\%) respondents were working at referral hospital, $73(24.5 \%)$ of them were working in Surgical ward, 213 (71.4\%) of respondents served less than 5years and more than three fourth of $226(75.6 \%)$ respondents attended their education at government institution (TABLE 1).

\begin{tabular}{|c|c|c|c|}
\hline Variables & Category & Frequency $(n=298)$ & Percentage (\%) \\
\hline \multirow{2}{*}{ Sex } & Male & 177 & 59.2 \\
\hline & Female & 121 & 40.8 \\
\hline \multirow{3}{*}{ Age } & $18-35$ & 57 & 19.0 \\
\hline & $36-55$ & 205 & 68.8 \\
\hline & $\geq 55$ & 36 & 12.2 \\
\hline \multirow{5}{*}{ Religion } & Orthodox & 128 & 42.8 \\
\hline & Muslim & 81 & 27.3 \\
\hline & Protestant & 77 & 25.9 \\
\hline & Catholic & 5 & 1.7 \\
\hline & Wakefata & 7 & 2.3 \\
\hline \multirow{3}{*}{ Educational level } & Diploma & 115 & 38.3 \\
\hline & Degree & 181 & 61.2 \\
\hline & Master & 2 & .7 \\
\hline \multirow{4}{*}{ Marital status } & Single & 146 & 48.8 \\
\hline & Married & 146 & 48.8 \\
\hline & Divorced/separated & 4 & 1.3 \\
\hline & Widowed & 2 & .7 \\
\hline \multirow{3}{*}{ Health facility } & Referral hospital & 236 & 79.2 \\
\hline & District hospitals & 62 & 20.8 \\
\hline & Total & 298 & 100 \\
\hline \multirow{9}{*}{ Working unit } & Medical & 64 & 21.5 \\
\hline & Surgical & 73 & 24.5 \\
\hline & ICU & 21 & 7.2 \\
\hline & Emergency & 33 & 11.1 \\
\hline & Gynecology and Obstetrics & 17 & 5.8 \\
\hline & Pediatrics & 38 & 12.8 \\
\hline & Psychiatrics & 9 & 3.1 \\
\hline & Other(Stroke,OR,NICU) & 42 & 14.0 \\
\hline & Total & 298 & 100 \\
\hline \multirow{4}{*}{ Years of experience } & $<5$ & 213 & 71.4 \\
\hline & $5-10$ & 72 & 24.2 \\
\hline & $>10$ & 13 & 4.4 \\
\hline & Total & 298 & 100 \\
\hline \multirow{3}{*}{ Training institution } & Government & 226 & 75.6 \\
\hline & Private & 72 & 24.4 \\
\hline & Total & 298 & 100 \\
\hline
\end{tabular}


- Pain management competency respondents related factor

One hundred eighty eight (63.1\%) have taken pre-service training. Only 138 (46.3\%) of respondents reported that they attended in-service training in the last six months. One hundred eighty eight (63.1\%) read book or journal on pain management and applied the knowledge they gained on pain management. Most of them 228 (76.5\%) had personally experienced pain which required to take medication and most of them 101(44.3\%) had mild pain at that times. Only $130(43.1 \%)$ of respondents reported that they had orientation on pain management as new staff during their starting patient pain management (TABLE 2).

Three constructs of Nurse-physician collaboration on pain management were identified: sharing of patient information on pain management, joint participation on pain management competency and degree of cooperation on pain management competency. Regarding joint participation on pain management competency 179 (60.1\%) respondents reported that had poor relationship on pain management competency (TABLE 3).

One hundred twenty nine (43.3\%) of respondents reported that hospitals provide continuous support and feedback on pain management at the hospital. Also one hundred fifty nine $(53.4 \%)$ respondents reported that the hospitals have pain management protocols. Only $(46.0 \%)$ of respondents reported that hospitals have guidelines on pain management at the hospital. Regarding frequency of read guidelines majority of them $95(32.2 \%)$ read always. More than half of $179(60.1 \%)$ of participants reported that pain medications are available at hospitals (TABLE 4).

- Proportion of respondents who correctly and in correctly respond to questions regarding nurses' pain management competency

Interpreting each specific item separately enabled the researcher to identify and to know areas where nurses' are not competent

\begin{tabular}{|c|c|c|c|}
\hline \multicolumn{2}{|l|}{ Characteristics } & \multirow{2}{*}{$\begin{array}{c}\text { Frequency } \\
188 \\
\end{array}$} & \multirow{2}{*}{$\begin{array}{c}\text { Percent } \\
63.1\end{array}$} \\
\hline Pre-service training on nain management & Yes & & \\
\hline Pre-service training on pain management & No & 110 & 36.9 \\
\hline \multirow{2}{*}{ In service training on pain management } & Yes & 138 & 46.3 \\
\hline & No & 160 & 53.7 \\
\hline \multirow{3}{*}{ Time when training taken } & In the last six month & 88 & 29.5 \\
\hline & One year ago & 28 & 9.4 \\
\hline & Before one year & 22 & 7.4 \\
\hline \multirow{2}{*}{ Read journal or book about pain management } & Yes & 188 & 63.1 \\
\hline & No & 110 & 36.9 \\
\hline \multirow{2}{*}{ Application of knowledge about pain in daily practice } & Yes & 160 & 53.4 \\
\hline & No & 28 & 9.7 \\
\hline \multirow{5}{*}{ provide care to patients' experiencing pain } & every1-2 hour & 103 & 34.6 \\
\hline & each shift & 145 & 48.6 \\
\hline & At least once week & 30 & 10.1 \\
\hline & Once month & 11 & 3.7 \\
\hline & Never & 9 & 3.0 \\
\hline \multirow{4}{*}{ Frequently using of objective tool while managing pain } & Never & 58 & 19.5 \\
\hline & Seldom & 63 & 21.1 \\
\hline & Some times & 119 & 39.9 \\
\hline & Always & 58 & 19.5 \\
\hline \multirow{2}{*}{ Personally Experienced pain which required to take medication } & Yes & 228 & 76.5 \\
\hline & No & 70 & 23.5 \\
\hline \multirow{3}{*}{ Perceived severity of experienced pain } & Mild & 101 & 44.30 \\
\hline & Moderate & 98 & 43.00 \\
\hline & Severe & 29 & 12.70 \\
\hline \multirow{2}{*}{ Orientation on pain management as new staff } & Yes & 130 & 43.10 \\
\hline & No & 168 & 56.90 \\
\hline
\end{tabular}




\begin{tabular}{|c|c|c|c|c|}
\hline \multicolumn{5}{|l|}{ Nurse-Physician relationship item } \\
\hline Joint participation on pain management competency & Rarely (n\%) & Sometimes (n\%) & Usually (n\%) & Always (n\%) \\
\hline $\begin{array}{l}\text { The nurses and the physicians have a mutual understanding of } \\
\text { the reasons for the change of pain management. }\end{array}$ & $60(20.1 \%)$ & $159(53.4 \%)$ & $46(15.4 \%)$ & $33(11.1 \%)$ \\
\hline $\begin{array}{l}\text { The nurses and the physicians check with each other concerning } \\
\text { whether a patient has any signs of side effects or complications } \\
\text { on pain management. }\end{array}$ & $58(19.5)$ & $131(44.0 \%)$ & $71(23.80)$ & $38(23.80 \%)$ \\
\hline $\begin{array}{l}\text { The nurses and the physicians share information about a } \\
\text { patient's reaction to explanations of his/her disease status and } \\
\text { treatment methods. }\end{array}$ & $55(18.50 \%)$ & $107(35.90 \%)$ & $94(31.50 \%)$ & $42(14.10 \%)$ \\
\hline $\begin{array}{l}\text { The nurses, the physicians, and the patient have the same } \\
\text { understanding of the patient's wish for cure and care. }\end{array}$ & $75(25.20 \%)$ & $122(40.90 \%)$ & $79(26.50 \%)$ & $22(7.40 \%)$ \\
\hline \multirow{2}{*}{$\begin{array}{l}\text { Over all Joint participation on pain management } \\
\text { competency }\end{array}$} & \multicolumn{2}{|l|}{ Good } & \multicolumn{2}{|l|}{ Poor } \\
\hline & \multicolumn{2}{|l|}{119 (39.9\%) } & \multicolumn{2}{|l|}{$179(60.1 \%)$} \\
\hline Sharing of patient information on pain management & Rarely (n\%) & Sometimes (n\%) & Usually (n\%) & Always (n\%) \\
\hline $\begin{array}{l}\text { The nurses and the physicians all know what has been explained } \\
\text { to a patient about his/her condition or treatment of pain. }\end{array}$ & $44(14.80 \%)$ & $144(48.30 \%)$ & $73(24.50 \%)$ & $35(12.40 \%)$ \\
\hline $\begin{array}{l}\text { The nurses and the physicians share information to verify the } \\
\text { effect of pain management. }\end{array}$ & $53(17.80 \%)$ & $124(41.60 \%)$ & $88(29.50 \%)$ & $33(11.10 \%)$ \\
\hline $\begin{array}{l}\text { The nurses and the physicians have the same understanding of } \\
\text { the future direction of the patient's pain management. }\end{array}$ & $75(25.20 \%)$ & $122(40.90 \%)$ & $79(26.50 \%)$ & $22(7.40 \%)$ \\
\hline $\begin{array}{l}\text { The nurses and the physicians identify the key person in pain } \\
\text { management. }\end{array}$ & $67(22.60 \%)$ & $109(36.70 \%)$ & $82(27.60 \%)$ & $39(13.10 \%)$ \\
\hline \multirow{2}{*}{ Over all Sharing of patient information on pain management } & \multicolumn{2}{|l|}{ Good } & \multicolumn{2}{|l|}{ Poor } \\
\hline & \multicolumn{2}{|l|}{134 (44.9\%) } & \multicolumn{2}{|l|}{$164(55.1 \%)$} \\
\hline Cooperativeness on pain management & Rarely (n\%) & Sometimes (n\%) & Usually (n\%) & Always (n \%) \\
\hline $\begin{array}{l}\text { The nurses and the physicians can easily talk about topics other } \\
\text { than topic related to pain management. }\end{array}$ & $89(30.10 \%)$ & $127(42.90 \%)$ & $56(18.90 \%)$ & $24(8.10 \%)$ \\
\hline $\begin{array}{l}\text { The nurses and the physicians can freely exchange information or } \\
\text { opinions about matters related to pain management. }\end{array}$ & $74(24.90 \%)$ & $138(46.50 \%)$ & $63(21.20 \%)$ & $22(7.40 \%)$ \\
\hline $\begin{array}{l}\text { The nurses and the physicians show concern for each other when } \\
\text { they are very tired on pain management. }\end{array}$ & $72(24.20 \%)$ & $125(41.90 \%)$ & $82(27.50 \%)$ & $19(6.40 \%)$ \\
\hline $\begin{array}{l}\text { The nurses and the physicians help each other on pain } \\
\text { management. }\end{array}$ & $60(20.10 \%)$ & $124(41.60 \%)$ & 77 (25.80\%) & $37(12.40 \%)$ \\
\hline \multirow{2}{*}{ Overall Cooperativeness on pain management } & \multicolumn{2}{|l|}{ Good } & \multicolumn{2}{|l|}{ Poor } \\
\hline & \multicolumn{2}{|l|}{$141(47.3 \%)$} & \multicolumn{2}{|l|}{$157(52.7 \%)$} \\
\hline \multirow{2}{*}{ Over all nurse-physician relationship } & \multicolumn{2}{|l|}{ Good } & \multicolumn{2}{|l|}{ Poor } \\
\hline & \multicolumn{2}{|l|}{$103(34.57 \%)$} & \multicolumn{2}{|l|}{195 (65.43\%) } \\
\hline
\end{tabular}

TABLE 4: Characteristics of organizational factor affecting respondents on pain management competency in Public hospitals Jimma zone, Oromia, March, 2017.

\begin{tabular}{|l|l|l|l|}
\hline \multicolumn{2}{|l}{ Organizational factors } & Frequency & Percent (\%) \\
\hline \multirow{2}{*}{ Hospitals provide continuous support and feedback } & Yes & 129 & 43.3 \\
\cline { 2 - 4 } & No & 169 & 56.7 \\
\hline \multirow{2}{*}{ Hospitals have pain management protocols } & Yes & 159 & 53.4 \\
\cline { 2 - 4 } & No & 139 & 46.6 \\
\hline \multirow{2}{*}{ Have Guidelines in Hospital } & Yes & 137 & 46.0 \\
\hline & No & 161 & 54.0 \\
\hline \multirow{3}{*}{ Frequency of Read guidelines } & Always & 32.2 \\
\cline { 2 - 4 } & Monthly & 31.9 \\
\cline { 2 - 4 } & Quarterly & 17 & 5.8 \\
\cline { 2 - 4 } & Yearly & 30.2 \\
\hline \multirow{2}{*}{ Pain medications are available } & Yes & 60.3 \\
\cline { 2 - 4 } & No & 179 & 39.7 \\
\hline
\end{tabular}


on pain management competency. Regarding multidimensional nature of pain 192 (64.8\%) respondents correctly identified that patients' spiritual beliefs may lead them to think pain and suffering are necessary. Only seventeen $(5.7 \%)$ respondents correctly answered on multidimensional nature of pain. From pain assessment and measurement only 112 (37.6\%) respondents correctly reported that Vital signs are always reliable indicators of the intensity of a patient's acute pain. Only $15(5.10 \%)$ respondents correctly answered on pain assessment and measurement. From clinical manifestation of pain one hundred sixty three $(54.7 \%)$ respondents correctly reported that respiratory depression rarely occurs in patients who have been receiving stable doses of opioids over a period of months. From the study it was indicated that on pain management majority $226(76.1 \%)$ of respondents correctly identified that the term 'equianalgesia' means approximately equal analgesia and is used when referring to the doses of various analgesics that provide approximately the same amount of pain relief and only $23.9 \%$ of respondents incorrectly

\section{(TABLE 5).}

\begin{tabular}{|c|c|c|c|}
\hline \multicolumn{2}{|l|}{ Variables } & Frequency & Percent \\
\hline \multicolumn{4}{|l|}{ Multidimensional nature of pain } \\
\hline \multirow{2}{*}{$\begin{array}{l}\text { Because their nervous system is underdeveloped, children under two years of age have } \\
\text { decreased pain sensitivity and limited memory of painful experiences. }\end{array}$} & Correct & 157 & 52.7 \\
\hline & Incorrect & 141 & 47.3 \\
\hline \multirow{2}{*}{ Patients who can be distracted from pain usually do not have severe pain. } & Correct & 164 & 55.0 \\
\hline & Incorrect & 134 & 45.0 \\
\hline \multirow{2}{*}{ Patients may sleep in spite of severe pain. } & Correct & 142 & 47.7 \\
\hline & Incorrect & 156 & 52.3 \\
\hline \multirow{2}{*}{ Patients' spiritual beliefs may lead them to think pain and suffering are necessary. } & Correct & 192 & 64.4 \\
\hline & Incorrect & 106 & 35.6 \\
\hline \multirow{2}{*}{$\begin{array}{l}\text { Narcotic/opioid addiction is defined as a chronic neuro biologic disease, characterized by } \\
\text { behaviors that include one or more of the following: impaired control over drug use, compulsive } \\
\text { use, continued use despite harm, and craving. }\end{array}$} & Correct & 187 & 62.8 \\
\hline & Incorrect & 111 & 37.2 \\
\hline \multirow{2}{*}{ Overall Multidimensional nature of pain } & Correct & 17 & 5.7 \\
\hline & Incorrect & 281 & 94.3 \\
\hline Pain assessment and measurement & & Frequency & Percent \\
\hline \multirow{2}{*}{$\begin{array}{l}\text { The best approach for cultural considerations in caring for patients in pain Patients should be } \\
\text { individually assessed to determine cultural influences. }\end{array}$} & Correct & 126 & 42.4 \\
\hline & Incorrect & 172 & 57.6 \\
\hline \multirow{2}{*}{ Vital signs are always reliable indicators of the intensity of a patient's acute pain. } & Correct & 112 & 37.6 \\
\hline & Incorrect & 186 & 62.4 \\
\hline \multirow{2}{*}{ Giving patients sterile water by injection (placebo) is a useful test to determine if the pain is real. } & Correct & 121 & 40.6 \\
\hline & Incorrect & 177 & 59.4 \\
\hline \multirow{2}{*}{$\begin{array}{l}\text { If the source of the patient's pain is unknown, opioids should not be used during the pain } \\
\text { evaluation period, as this could mask the ability to correctly diagnose the cause of pain. }\end{array}$} & Correct & 124 & 41.6 \\
\hline & Incorrect & 174 & 58.4 \\
\hline \multirow{2}{*}{ The most accurate judge of the intensity of the patient's pain is the patient. } & Correct & 138 & 45.6 \\
\hline & Incorrect & 160 & 54.4 \\
\hline \multirow{2}{*}{$\begin{array}{l}\text { Sedation assessment is recommended during opioid pain management because excessive } \\
\text { sedation precedes opioid-induced respiratory depression. }\end{array}$} & Correct & 211 & 71.0 \\
\hline & Incorrect & 87 & 29.0 \\
\hline \multirow{2}{*}{$\begin{array}{l}\text { Children less than } 11 \text { years old cannot reliably report pain so clinicians should rely solely on the } \\
\text { parent's assessment of the child's pain intensity. }\end{array}$} & Correct & 140 & 47.1 \\
\hline & Incorrect & 158 & 52.9 \\
\hline \multirow{2}{*}{$\begin{array}{l}\text { Patient A: Andrew is } 25 \text { years old and this is his first day following abdominal surgery. As } \\
\text { you enter his room, he smiles at you and continues talking and joking with his visitor. Your } \\
\text { assessment reveals the following information: } B P=120 / 80 ; H R=80 ; R=18 ; \text { on a scale of } 0 \\
\text { to } 10(0=\text { no pain/discomfort, } 10=\text { worst pain/discomfort) he rates his pain as } 8 \text {. A. On the } \\
\text { patient's record you must mark his pain on the scale below. Circle the number that represents } \\
\text { your assessment of Andrew's pain(8).. }\end{array}$} & Correct & 72 & 23.7 \\
\hline & Incorrect & 226 & 75.3 \\
\hline \multirow{2}{*}{$\begin{array}{l}\text { Patient B: Robert is } 25 \text { years old and this is his first day following abdominal surgery. As you } \\
\text { enter his room, he is lying quietly in bed and gri-maces as he turns in bed. Your assessment } \\
\text { reveals the following information: BP }=120 / 80 ; \mathrm{HR}=80 ; \mathrm{R}=18 ; \text { on a scale of } 0 \text { to } 10(0=\text { no } \\
\text { pain/ discomfort, } 10=\text { worst pain/discomfort) he rates his pain as } 8 \text {. A. On the patient's record } \\
\text { you must mark his pain on the scale below. Circle the number that represents your assessment } \\
\text { of Robert's pain: (8) }\end{array}$} & Correct & 89 & 29.9 \\
\hline & Incorrect & 209 & 70.1 \\
\hline
\end{tabular}




\begin{tabular}{|c|c|c|c|}
\hline \multirow{2}{*}{ Over all Pain assessment and measurement } & Correct & 15 & 5.10 \\
\hline & Incorrect & 283 & 94.90 \\
\hline Clinical manifestation of pain & & Frequency & Percent \\
\hline \multirow{2}{*}{$\begin{array}{l}\text { Respiratory depression rarely occurs in patients who have been receiving stable doses of } \\
\text { opioids over a period of months. }\end{array}$} & Correct & 163 & 54.7 \\
\hline & Incorrect & 135 & 45.3 \\
\hline \multirow{2}{*}{$\begin{array}{l}\text { A patient with persistent cancer pain has been receiving daily opioid analgesics for } 2 \text { months. } \\
\text { Yesterday the patient was receiving morphine } 200 \mathrm{mg} / \text { hour intravenously. Today he has } \\
\text { been receiving } 250 \mathrm{mg} / \text { hour intravenously. The likelihood of the patient developing clinically } \\
\text { significant respiratory depression in the absence of new co morbidity is }>1 \% \text {. }\end{array}$} & Correct & 96 & 32.2 \\
\hline & Incorrect & 202 & 67.8 \\
\hline \multirow{2}{*}{$\begin{array}{l}\text { Following abrupt discontinuation of an opioid, physical dependence is manifested sweating, } \\
\text { yawning, diarrhea and agitation with patients when the opioid is abruptly discontinued. }\end{array}$} & Correct & 91 & 30.6 \\
\hline & Incorrect & 207 & 69.4 \\
\hline \multirow{2}{*}{$\begin{array}{l}\text { True regarding opioid induced respiratory depression Obstructive sleep apnea is an important } \\
\text { risk factor. }\end{array}$} & Correct & 119 & 40.2 \\
\hline & Incorrect & 179 & 59.8 \\
\hline \multirow{2}{*}{ Over all clinical condition } & Correct & 16 & 5.36 \\
\hline & Incorrect & 282 & 94.64 \\
\hline Pain management & & Frequency & Percent \\
\hline \multirow{2}{*}{$\begin{array}{l}\text { Aspirin and other nonsteroidal anti-inflammatory agents are not effective analgesics for painful } \\
\text { bone metastases. }\end{array}$} & Correct & 160 & 53.7 \\
\hline & Incorrect & 138 & 46.3 \\
\hline \multirow{2}{*}{$\begin{array}{l}\text { Combining analgesics that work by different mechanisms may result in better pain control with } \\
\text { fewer side effects than using a single analgesic agent. }\end{array}$} & Correct & 168 & 56.4 \\
\hline & Incorrect & 130 & 43.6 \\
\hline \multirow{2}{*}{ The usual duration of analgesia of 1-2 mg morphine IV is 4-5 hours. } & Correct & 124 & 41.6 \\
\hline & Incorrect & 174 & 58.4 \\
\hline \multirow{2}{*}{ Opioids should not be used in patients with a history of substance abuse. } & Correct & 138 & 46.3 \\
\hline & Incorrect & 160 & 53.7 \\
\hline \multirow{2}{*}{ Elderly patients cannot tolerate opioids for pain relief. } & Correct & 153 & 51.3 \\
\hline & Incorrect & 145 & 48.7 \\
\hline \multirow{2}{*}{ Patients should be encouraged to endure as much pain as possible before using an opioid. } & Correct & 155 & 52.0 \\
\hline & Incorrect & 143 & 48 \\
\hline \multirow{2}{*}{$\begin{array}{l}\text { After an initial dose of opioid analgesic is given, subsequent doses should be adjusted in } \\
\text { accordance with the individual patient's response. }\end{array}$} & Correct & 191 & 64.1 \\
\hline & Incorrect & 107 & 35.9 \\
\hline \multirow{2}{*}{$\begin{array}{l}\text { Anticonvulsant drugs such as gabapentin (Neurontin) produce optimal pain relief after a single } \\
\text { dose. }\end{array}$} & Correct & 139 & 46.6 \\
\hline & Incorrect & 159 & 53.4 \\
\hline \multirow{2}{*}{$\begin{array}{l}\text { Benzodiazepines are not effective pain relievers and are rarely recommended as part of an } \\
\text { analgesic regiment. }\end{array}$} & Correct & 166 & 55.7 \\
\hline & Incorrect & 132 & 44.3 \\
\hline \multirow{2}{*}{$\begin{array}{l}\text { Vicodin (hydrocodone } 5 \mathrm{mg}+\text { acetaminophen } 300 \mathrm{mg} \text { ) PO is approximately equal to } 5-10 \mathrm{mg} \\
\text { of morphine PO. }\end{array}$} & Correct & 165 & 55.4 \\
\hline & Incorrect & 133 & 44.6 \\
\hline \multirow{2}{*}{$\begin{array}{l}\text { The term 'equianalgesia' means approximately equal analgesia and is used when referring to the } \\
\text { doses of various analgesics that provide approximately the same amount of pain relief. }\end{array}$} & Correct & 226 & 76.1 \\
\hline & Incorrect & 72 & 23.9 \\
\hline \multirow{2}{*}{$\begin{array}{l}\text { The recommended route of administration of opioid analgesics for patients with persistent } \\
\text { cancer-related pain is oral. }\end{array}$} & Correct & 63 & 21.1 \\
\hline & Incorrect & 235 & 78.9 \\
\hline \multirow{2}{*}{$\begin{array}{l}\text { The recommended route administration of opioid analgesics for patients with brief, severe pain } \\
\text { of sudden onset such as trauma or postoperative pain is Intravenous. }\end{array}$} & Correct & 186 & 62.4 \\
\hline & Incorrect & 112 & 37.6 \\
\hline \multirow{2}{*}{$\begin{array}{l}\text { The drug of choice for the treatment of prolonged moderate to severe pain for cancer patients } \\
\text { is morphine. }\end{array}$} & Correct & 178 & 60.1 \\
\hline & Incorrect & 110 & 39.9 \\
\hline \multirow{2}{*}{$\begin{array}{l}\text { The most likely reason a patient with pain would request increased doses of pain medication is } \\
\text { patient is experiencing increased pain. }\end{array}$} & Correct & 122 & 40.9 \\
\hline & Incorrect & 176 & 59.1 \\
\hline & Correct & 46 & 15.4 \\
\hline & Incorrect & 252 & 84.6 \\
\hline he drug that is useful for treatment of cancer pain is Ibuprofen.Hydromorhone,Gabapentir & Correct & 115 & 38.6 \\
\hline 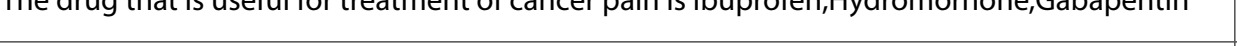 & Incorrect & 183 & 61.4 \\
\hline Patient whe develon nain already have an alcohol and/or drug abuce nrok & Correct & 145 & 48.8 \\
\hline . & Incorrect & 153 & 51.2 \\
\hline he time to peak effect for morphine qiven IV is $15 n$ & Correct & 177 & 59.6 \\
\hline & Incorrect & 121 & 40.1 \\
\hline
\end{tabular}




\begin{tabular}{|c|c|c|c|}
\hline \multirow{2}{*}{$\begin{array}{l}\text { Your assessment, Andrew, is made two hours after he received morphine } 2 \text { mg IV. Half hourly } \\
\text { pain ratings following the injection ranged from } 6 \text { to } 8 \text { and he had no clinically significant } \\
\text { respiratory depression, sedation, or other untoward side effects. He has identified } 2 / 10 \text { as an } \\
\text { acceptable level of pain relief. His physician's order for analgesia is "morphine IV } 1-3 \mathrm{mg} \text { q1h } \\
\text { PRN pain relief." Check the action you will take at this time. (Administer morphine } 3 \text { mg IV now) }\end{array}$} & Correct & 69 & 23.2 \\
\hline & Incorrect & 229 & 76.8 \\
\hline \multirow{2}{*}{$\begin{array}{l}\text { Your assessment, Robert, is made two hours after he received morphine } 2 \mathrm{mg} \text { IV. Half hourly } \\
\text { pain ratings following the injection ranged from } 6 \text { to } 8 \text { and he had no clinically significant } \\
\text { respiratory depression, sedation, or other untoward side effects. He has identified } 2 / 10 \text { as an } \\
\text { acceptable level of pain relief. His physician's order for analgesia is "morphine IV } 1-3 \mathrm{mg} \text { q } 1 \mathrm{~h} \\
\text { PRN pain relief." Check the action you will take at this time: (Administer morphine } 3 \mathrm{mg} \text { IV now) }\end{array}$} & Correct & 65 & 21.8 \\
\hline & Incorrect & 233 & 71.2 \\
\hline \multirow{2}{*}{ Overall pain management } & Correct & 5 & 1.68 \\
\hline & Incorrect & 293 & 98.32 \\
\hline \multirow{2}{*}{ Over all pain management domains } & Correct & 19 & 6.37 \\
\hline & Incorrect & 289 & 93.63 \\
\hline
\end{tabular}

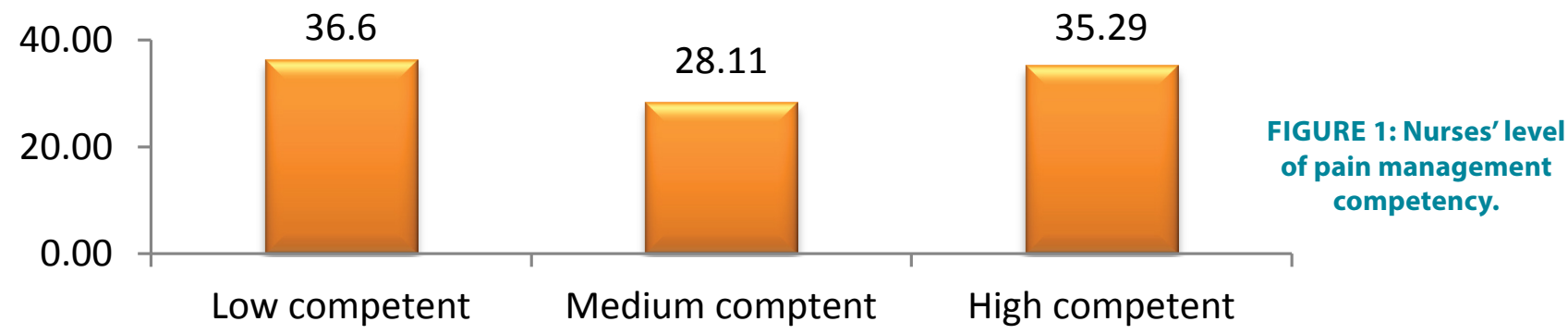

\begin{tabular}{|c|c|c|c|c|c|c|c|c|c|c|}
\hline Variable & Catea & & npete & lev & & & R(05\%CI) & & $A O R(95 \% C I)$ & P-value \\
\hline valiavie & carey & & tent & & petent & & (ו) & & (5) & -value \\
\hline Preservice training & Yes & & 9\%) & & $3.13 \%)$ & 1.63 & $(0.98,2.75)$ & 1.27 & $7(0.707,2.28)^{* *}$ & 0.42 \\
\hline on pain & No & & & & $75 \%)$ & & 1 & & 1 & \\
\hline Have you personal & Yes & & $6 \%)$ & & (61.4) & 1.93 & $(1.04,3.55) *$ & & $.87(0.97,3.59)$ & 0.061 \\
\hline experience pain & No & & $0 \%)$ & & $7.9 \%)$ & & 1 & & 1 & \\
\hline Orientation on & Yes & & $9 \%)$ & & $9.1 \%)$ & 1.73 & $(1.06,2.81)^{*}$ & & $.52(0.88,2.62)$ & .0 .132 \\
\hline pain management & No & & $2 \%)$ & & $9.8 \%)$ & & 1 & & 1 & \\
\hline Read Guidelines on & Yes & & $4 \%)$ & & $0.6 \%)$ & 0.71 & $(0.42,1.21)^{*}$ & & $.63(0.35,1.12)$ & 0.12 \\
\hline pain & No & & $8 \%)$ & & $58.2 \%)$ & & 1 & & 1 & \\
\hline Dratoral facility & yes & & $9 \%)$ & & $9.1 \%)$ & 1.7( & $(1.04,2.75) *$ & & $59(0.908,2.81)$ & 0.104 \\
\hline Protocom tacinty & no & & $2 \%)$ & & $9.8 \%)$ & & 1 & & 1 & \\
\hline & Medical & & $3 \%)$ & & $6,7 \%)$ & 2.01 & $(1.04,3.85) *$ & & $5(1,02,4.12) * *$ & 0.044 \\
\hline Working Unit & surgical & & $2 \%)$ & & $.28 \%)$ & 0.27( & $(0.085,0.83) *$ & & $19(0.05,0.64) *$ & 0.007 \\
\hline & Maternal h & alth & $2 \%)$ & & $.88 \%)$ & & 1 & & 1 & \\
\hline Nurse-Physician wo & rk relation & ip c & ment & pe & & & & & & \\
\hline Nurse-physician rela & ationship & Cat & Com & & Incom & betent & COR & & AOR & P-Value \\
\hline Overall & & Go & $70(4$ & & $89(56$ & & $2.34(1.04,3$. & 38) * & $2.36(1.36,4.08) * *$ & 0.005 \\
\hline Over dil & & Poc & $32(2$ & & $95(74$ & & 1 & & 1 & \\
\hline
\end{tabular}

The result indicates that majority 189 (63.4\%) of respondents were not competent on pain management competency and only 109 (36.6\%) are competent on pain management competency. The FIGURE 1 below indicates nurses' level of pain management competency.
A significance level of $\mathrm{P}<0.007$ was accepted as statistically significant (TABLE 6).

\section{Discussion}

This research is the first to provide data on the level of pain management competency among nurses' working in Jimma public 
hospitals, Jimma zone, Oromia regional state. The tool used in this present study has also been utilized in international studies with nurses and anesthetists from a variety of clinical backgrounds.

In this study effort has been made to identify level of nurses' pain management competency. The study indicated that the overall $36.6 \%$ of the study respondents were competent on pain management. This finding is lower as compared to the study conducted in Gondar referral hospital on competency nurses' patient management which was (48.7\%) (32). This might be due to lack of training on pain management.

The study revealed that $55.0 \%$ patients who can be distracted from pain usually do not have severe pain. This figure is high as compared to the study conducted in Bangladesh in which $23.7 \%$ nurses reported that patients who can be distracted from pain usually do not have severe pain [17]. This might be due to the fact that hospital had protocol. In this study $51.3 \%$ nurses' reported that elderly patients cannot tolerate opioids for pain relief which is lower as compared to the study conducted in Gardnerhospital which indicated that $90.2 \%$ respondents correctly answered that elderly patients cannot tolerate opioids for pain relief [18]. This might be nurses' were not knowledgeable pain medication that was indicated for elderly patients. Nurses' in this study reported that $64.4 \%$ patients' spiritual beliefs may lead them to think pain and sufferings are necessary. This figure is lower as compared to the study conducted in Bangladesh which was $80.9 \%$ [17]. This discrepancy might be because of nurses' lack patients' spiritual belief about pain management. Hence, the hospital needs to work to increase the cultural competency of nurses.

This study revealed that $37.6 \%$ of nurses' said Vital signs are always reliable indicators of the intensity of a patient's pain which is high as compared to $26.9 \%$ in Bangladesh [17]. The finding underscores that nurses' know more as vital sign are not reliable indicators of the patient assessment and measurement. In our finding $41.6 \%$ of nurses said that if the source of the patient's pain is unknown, opioids should not be used during the pain evaluation period, as this could mask the ability to correctly diagnose the cause of pain. This finding is low as compared to study conducted in Bangladesh in which $53.2 \%$ gave correct answer for the same question [17].
This study finding also showed that nurses' need to know pain evaluation period after source has been identified. The finding also indicated that $45.6 \%$ respondents correctly answered that the most accurate judge of the intensity of the patient's pain is the patient This figure is high as compared to the study conducted in Hong kong in which $36.3 \%$ respondents reported that the most accurate judge of the intensity of the patient's pain is the patient (19). This might be due to nurses' awareness on assessment of pain management.

The study indicated that $41.1 \%$ respondent correctly answered the usual duration of 1-2 $\mathrm{mg}$ morphine IV is $4-5$ hours. . This study is high when compared to the study conducted in Bangladesh in which 34\% of respondents agree that the usual duration of analgesia of 1-2 $\mathrm{mg}$ of morphine intravenous IV is 4-5 hourly [17]. The finding underscores that nurses' need to know the usual duration of analgesia administration. In our study, $40.6 \%$ of nurses responded affirmatively to a question "Giving patients sterile water by injection (placebo) is a useful test to determine if the pain is real". The finding is much better than the finding of study conducted in Bangladesh, in which 81.9\% did not support giving patients sterile water by injection (placebo) to determine if the pain is real [17]. This indicated that nurses' need to know real pain testing method. The result also indicated that $47.1 \%$ Children less than 11 years old cannot reliably report pain so clinicians should rely solely on the parent's assessment of the child's pain intensity. The finding is low as compared to the study conducted in Hong Kong in which $63.7 \%$ respondent reported that Children less than 11 years old cannot reliably report pain so nurses should rely solely on the parent's assessment of the child's pain intensity [19]. Because Nurses' do not believe pain reported by children because they might think that children's understanding of pain is limited.

The respondents reported that $54.7 \%$ respiratory depressions rarely occurs in patients who have been receiving stable doses of opioids over a period of months. It is consistent with study done in Bangladesh it was $54.3 \%$ believed that respiratory depression would occur in patients receiving who have been receiving stable doses of opioids over a period of months [17]. The study revealed that, from the two patient case studies were used to determine nurses' ability to make decisions correctly about pain assessment data and interventions. Results 
showed that nurses might assess the pain score of a grimacing patient in comparison with a smiling patient as indicative of increased pain even though both patients reported the same pain score. Results revealed that nurses gave a lower pain score to the smiling patient as compared with the grimacing patient and administered less dose of analgesia to the smiling patient. Thus, more nurses correctly assessed pain when the patient grimaced than when the patient was smiling. The study revealed that nurses relied on patient's appearance in assessing the pain and not depending on patients' statements. Unfortunately, only $23.2 \%$ of nurses' in the first case study and $21.8 \%$ in the second case study indicated they would administer the recommended amount of morphine on the basis of the assessment data. This finding is high as compared to the study conducted in Jordanian, $6.7 \%$ in the first case of patient and $10 \%$ in the case of second patient [20]. One possible explanation of this finding is that nurses' were not adequately trained to understand the concentration of opioid doses and its administration.

In our study, 53.4\% respondents reported that the hospitals have pain management protocols. This figure is higher when compared to the finding of the study conducted in Bangladesh, in which $18.3 \%$ of the nurses stated that there was a pain management standard or protocol in their hospital [26]. This may increase nurse pain management competency. Also $46.0 \%$ of our respondents reported that the hospital has guidelines on pain management. This finding is lower when compared to the study conducted in Uganda, in which majority of respondents reported that their hospital has /guidelines which was (78.8\%) [19]. The presence of guideline may affect nurses' competency of pain management. In the present study $60.1 \%$ of study participants reported that pain medications was available at hospitals. It was inconsistent with $\mathrm{WHO}$ 2006 report on Ethiopian morphine per capita consumption which found $0.0002 \mathrm{mg} /$ capita. However, it was lower when compared to global mean of $5.85 \mathrm{mg}$ [29]. For this study we need to have follow up research for nurses' pain management competency. There was significant association between those who had pain management protocol than those who hadn't pain management protocol on pain management competency. Those hospitals need to have pain management protocol.
The finding indicated that $46.3 \%$ of respondents reported that they had in-service training on pain management competency. This figure is lower when compared to the study conducted in Ugandan and Irish studies in which $69 \%$ and $62.8 \%$ reported that they had in-service training on pain management $[19,26]$. This implies that the majority of nurses' in our study didn't get training regarding pain management competency. The hospital, therefore, needs to give in-service training for nurses' regarding competency of nurses' pain management. Nurses' those who rated the overall nurse-physician relationship as good are 2.4 times more likely competent on hospitalized patient pain management than those who rated as poor with. Respondents who were working at medical two times more competent on patient pain management than maternal ward. This might be medical ward is critical area and well trained nurses' assigned to medical ward. Nurses' who were working at surgical wad $81 \%$ less likely competent on patient pain management than maternal ward. This might be Surgical ward is critical working area and well trained nurses' assigned to surgical ward.

The finding indicated that $76.5 \%$ respondents reported that they had personally experienced pain which required taking medication. This finding is higher compared to North-eastern united states indicated that $64.5 \%$ who answered yes to having had past personal pain experience that required medication or treatment [23]. This might be due to nurses' work busy. It increases their competency of pain management.

There is no significant association between age of respondents, working area of respondents, sex, year of patient care and in-service training.

\section{Conclusion and Recommendation}

The overall nurse' pain management competency level in Jimma zone, Public hospital is very low. Among many factors contributed to the nurses' patient pain management competency is nurse-physician work relationship, hospital having protocol and working unit. Nurse related factor like using objective tool on pain management, read any book or journal about pain management, had association with nurse pain management competency.

The researcher recommends having future research nurses' competency of pain 
management. The hospital need to provide training for nurses', using objective tool on pain management competency and reading journal or books about pain for nurse on pain management is the most important for improvement of nurses' competency on pain management. Those show that Jimma public hospitals should design strategy to improve pain management competency. Nurse pain management competency enhances utilization of protocols, that specifies pain management by unit and promote nurse-physician relation.

This research initiates another preferably observational study in a greater number of clinical settings with a larger sample size to enhance generalizability.

\section{Conflict of Interests}

All authors declared that they have no conflict of interests. Jimma University covered only the survey cost for this study and there is no any funding organization.

\section{Authors' Contribution}

Gugsa Nemera and Abiru Neme conceived and designed the protocol. Abiru Neme, contributed on data analysis, and checked the draft. Abiru Neme and Gadisa Bekele prepared manuscript. All authors read and approved the final paper.

\section{Acknowledgements}

We would like to thank the Jimma zone administrative as well as the administrative staffs for all the help and support given for us during the data collection period. We also thank Jimma university institute of health for permitting us to conduct this study. 


\section{References}

IASP. International association for the study of pain. IASP pain terminology. 13(12), 9-111 (2010).

Lilley L, Collins R, Harrington S, et al. Pharmacology and the nursing process. 6th edition. St Louis: Mosby Elsevier. 21(13), 7-133 (2011).

Long CO, Morgan BM. Clinical competency in pain management for home health nurses and hospice nurses. In C J Humphrey (Ed.), Pain management the resource guide for home health and hospice nurses, baltimore, hopkins medical products. 18(13), 9-187 (2008).

Berman A, Snyder SJ, Kozier B, et al. Kozier and Erbs's fundamentals of nursing: concepts process and practice. $8^{\text {th }}$ edition. Upper Saddle River, NJ: Pearson. 16(7), 5-121 (2008).

Koebner IJ, Herr K. Adopting pain management competencies. Pain Med. 14(7), 964-965 (2013).

Watt WJ, Siddall PJ. Improving pain practices through core competencies. Pain Med. 14(7), 966-967 (2013).

Kizer KW. Advancing pain care core competencies for pain management. Pain Med. 14(7), 962-963 (2013).

Walsh NE, Brooks P, Meike HJ, et al. Standards of care for acute and chronic musculoskeletal pain: The bone and joint decade. Arch Phys Med Rehabil. 89(13), 45-1830 (2008).

Wells N, Pasero C, Mc Caffery M. Patient safety and quality: An evidence-based handbook for nurses. Rockville, MD: Agency for healthcare research and quality. 23(6), 9-134 (2011).

Fine PG The use of opioids in pain management. The academy for continued healthcare learning. 30(6), 8-154 (2012).

Innis J, Bikaunieks N, Petryshen P, et al. Patient satisfaction and pain management: an educational approach. J qual. 19(4), 322-327 (2004).

Classification of pain-pain management. 24(7), 7-133 (2010).

Ubino $\mathrm{V}$. The nurses role in the postoperative pain management. Minerva Chirurgica. 58(6), 73-869.

Brockopp DY, Brockopp G, Warden S, et al.
Barriers to change: a pain management project. Int J Nurs Stud. 35(6), 226-232 (2011).

International association of the study of pain. sub-committee on taxonomy. Pain terms: a list with definitions and notes on usage. Pain. 22(6), 52-249 (2012).

Mezey, M. Assessing pain as the fifth vital sign in ling-term care facilities: Recommendations from the field. J Nurs. 31(7), 16-24 (2005).

Hossain, Sazzad, Wiroonpanich, et al. Attitudes and pain management practice of post-operative children in Bangladesh. 21(7), 9-21 (2014).

Sanna S. Nurses knowledge about pharmacological and nonpharmacological pain management in children. J Pain Symptom Manage. 18(4), 99-289 (2009).

Lui LY, So WK, Fong DY. Knowledge and attitudes regarding pain management among nurses in Hong Kong medical units. J Clin Nurs. 17(7), 21-18 (2014).

Mahmoud H, Muhammad D. Knowledge and attitudes regarding pain management among Jordanian nurses. 8(5), 6-20 (2013).

Liou SR, Chang CH, Tsai HM, et al. The effects of a deliberate practice program on nursing students perception of clinical competence. Nurs Educ. (33), 358-363 (2012).

Nurse-Physician collaboration scale: development and psychometric testing. $J A d v$ Nurs. 65(7), 1497-1508 (2013).

Brown CA, Richardson C. Nurses in the multi-professional pain team: A study of attitudes, beliefs and treatment endorsements. Eur J Pain. 10(3), 13-22 (2013).

Berry PH, Dahl JL. The new JCAHO pain standards: Implications for pain management nurses. Pain Manag Nurs. (1), 3-12 (2012).

Wessman AC. Nurses personal pain experiences and their pain management knowledge. J Contin Educ Nurs. 30(4), 7-152 (2010).

Kizza, Irene Betty, Nurses knowledge and practices related to pain assessment in critically ill patients at Mulago Hospital, Uganda: Muhimbili University of Health and Allied Sciences. 23(11), 11-112 (2012).

Ireland Pain Society Nurses' Interest Group, Ireland Pain Management Nursing Knowledge 
and Skills Framework for Registered Nurses. 25(17), 11-123 (2013).

Idang N, Ojong MM, Ojong A et al. Nurses' assessment and management of pain among surgical patients in secondary health facility in Calabar Metropolis, Cross River State, Nigeria. Eur J Exp Bio. 4(1), 20-315 (2014).

Vickers, Niamh. Knowledge and attitudes regarding pain among surgical nurses in three teaching hospitals in Ireland: Dublin City University. 21(6), 9-132 (2011).

Abrahamson K, Fox R, Doebbeling B. Original research: Facilitators and barriers to clinical practice guideline use among nurses. $A m$ J Nurs. 24(11), 26-35 (2012).

Palliative care and pain relief Special-Issue. 21(5), 7-140 (2011).

Klein CJ, Fowles ER. An investigation of nursing competence and the competency outcomes performance assessment curricular approach: senior students' self-reported perceptions. 21(8), 7-121 (2015).

Knowledge and attitudes survey regarding pain" developed by Betty Ferrell and Margo McCaffery. 23(9), 3-131 (2014).

https://en.wikipedia.org/wiki/Jimma 